\title{
Interpretations Outcomes of Assays Fulfilling on Knockdown Cells and in Vivo
}

\author{
Ponizovskiy MR* \\ Head of Laboratory Biochemistry and Toxicology, Germany
}

*Corresponding author: Ponizovskiy MR, Head of Laboratory Biochemistry and Toxicology, Germany.

\begin{abstract}
The interpretation the experimental assays on „Knockdown cells“ should be limited considering interactions between each cell and an organism if the experimental assays would be carried out „in vivo“.
\end{abstract}

\section{Interdependence Knockdown Cells and in vivo}

Just obligatory interactions between extracellular medium and intracellular medium are determined by interactions between intracellular balance anabolic processes \& catabolic prosesses and extracellular balance anabolic processes \& catabolic prosesses which induce fluctuating balance inner chemical potential cells $\left(\mu_{\text {inner cell }}\right) \&$ outer chemical potential cells $\left(\mu_{\text {outer cell }}\right)$. The fluctuating balance inner chemical potential cells $\left(\mu_{\text {inner cell }}\right)$ \& outer chemical potential cells $\left(\mu_{\text {outer cell }}\right)$ exerts interaction between intracellular medium and extracellular medium which is the driving mechanism as of cellular development as well as of activity cellular cycle in norm and in pathology [1-4]. Besides extracellular medium of each cell is the Internal Medium of an organism containing respiratory electron transport chain with its NADH-Q oxidoreductase of Complex $\rightarrow$ Succinate- $Q$ oxidoreductase via flavin adenine dinucleotide (FAD) coenzyme of Complex II $\rightarrow$ Q-cytochrome c oxidoreductase of Complex III $\rightarrow$ Cytochrome c oxydase of Complex IV $\rightarrow$ ATP synthase for oxidative phosphorylation of Complex $\mathrm{V}$ as well as Reactive Oxygen Species (ROS), superoxide $\left(\mathrm{O}_{2}^{*}\right)$, Free Radicals operations and so on, i.e. oxidative processes from lung respiratory processes through oxyhemoglobin in erythrocytes and further respiratory electron transport chain into extracellular medium forming balance anabolic processes \& catabolic prosesses which cause outer chemical potential cells $\left(\mu_{\text {outer cell }}\right)$ [5-8]. Thus revealing some Factors can be as Products of some biochemical Reactions as well as Reagents of these Reactions although Reactant of chemical reaction can be transited into Product of this reaction and vice versa which depends on changes balance inner chemical potential cells $\left(\mu_{\text {inner cell }}\right)$ \& outer chemical potential cells $\left(\mu_{\text {outer cell }}\right)$ according Henderson - Has selbach formula, for example, for reaction $\mathrm{A}+\mathrm{B}=\mathrm{C}+\mathrm{D}$. (A and B are Reactants; $\mathrm{C}$ and $\mathrm{D}$ are Products; $\mathrm{K}$ is Constant of Reaction):

$$
K=\frac{[C] \times[D]}{[A] \times[B]}
$$

Therefore the experimental assays on „Knockdown cells" give possibility to reveal different Factors, Kinases and the other cellular elements and to determine empirical their cellular or local influences on cellular activity. However experimental assays on „Knockdown cells" don't give possibility to explain mechanisms of these Factors action in cellular cycle and mechanisms influences on an organism's metabolism and ist development. Just study interactions intracellular medium with internal medium of an organism as extracellular medium explain mechanisms of these Factors action in cellular cycle and mechanisms influences on an organism's metabolism and ist development [9-14]. This interactions between intracellular medium and internal medium of an organism as extracellular medium can been determined either through consideration these interactions from the point of views of thermodynamics [its laws], biophysics and biochemistry or via supplemental experimentals in vivo.

\section{Acknowledgement}

None.

\section{Conflict of Interest}

No conflict of interest. 


\section{References}

1. Ponizovskiy MR (2017) Mechanisms of cancer growth and metastasis with substantiation of new methods cancer therapy. Molecular medicine: Current Aspects 1(1): 1-12.

2. Ponizovskiy MR (2018) The mechanism of cancer cellular genome disorder and comparison therapeutic effects of modern methods and new method cancer treatment. Journal of Genetic Disorders 2(8): 1-17.

3. Ponizovskiy MR (2016) Role of Krebs cycle in mechanism of stability Internal Medium and Internal Energy in an organism in norm and in mechanism of cancer pathology, Modern chemistry \& Applications 4(4): 1-8.

4. Ponizovskiy MR (2017) Mechanisms of changes balance anaerobic processes and aerobic processes in cancer metabolism causing Warburg effect mechanism. Journal of biomolecular research \& Therapeutics 6(1): 1-9.

5. Ponizovskiy MR (2013) Biophysical and biochemical transmutation of mitochondrial function in cancer genesis, Biochemistry \& Analytical Biochemistry 2(3): 1-9.

6. Furda Amy Marie (2011) the role of mtDNA damage in mitochondrial disfunction, University Pitstsburg 145.

7. Frohe L (1982) Free radicals in biology, in Pryor WA (ed), Academic Press, NewYork, USA, pp. 223-275.
8. Emanuel NM (1977) Kinetics experimental tumorous processes, Moscow Science 419.

9. Ponizovskiy MR (2015) Biophysical and Biochemical Mechanisms of Interactions Cytoplasm Processes with Nucleus Processes and Mitochondria Processes in Norm and in Pathology. Journal of Molecular and Genetic Medicine 9(3): 1-13.

10. Ponizovskiy MR (2017) Mechanisms of cancer growth and metastasis with substantiation of new methods cancer therapy. Molecular medicine: Current Aspects 1(1): 1-12.

11. Ponizovskiy MR (2019) A newer method cancer treatment which is based on Link Rearrangement Operations of $\mathrm{T}$ cells. International journal of Cancer and Oncology 6(2): 42-51.

12. Ponisovskiy MR (2010) Cancer metabolism and the Warburg effect as anabolic process outcomes of oncogene operation. Critical Reviews in Eukaryotic Gene Expression 20(4): 325-339.

13. Ponizovskiy MR (2017) Mechanisms of cancer growth and metastasis with substantiation of new methods cancer therapy. Molecular medicine: Current Aspects 1(1): 1-12.

14. Ponizovskiy MR (2018) The mechanism of cancer cellular genome disorder and comparison therapeutic effects of modern methods and new method cancer treatment. Journal of Genetic Disorders 2(8): 1-17. 